\title{
Mohammed bin Salman: legitimidad y nuevos desafíos*
}

\author{
Ignacio Teruel** \\ Universidad de Congreso, Argentina \\ https://doi.org/10.15446/frdcp.n21.93768
}

\section{Resumen}

El presente artículo tiene por objetivo analizar la legitimidad del príncipe heredero de Arabia Saudita, Mohammed bin Salman, considerando los pilares históricos de legitimación de la corona y su situación actual. La investigación fue abordada desde un enfoque cualitativo y se utilizó el método hipotético-deductivo, formulando una pregunta guía de la investigación y estableciendo, a partir de ella, los objetivos y la hipótesis del trabajo, la cual será comprobada utilizando la reflexión racional y la observación de la realidad, mediante un análisis documental de libros impresos y digitales, notas periodísticas, publicaciones en revistas electrónicas e informes gubernamentales. Dicha hipótesis es que la legitimidad de Mohammed bin Salman se encuentra debilitada a causa de su creciente personalismo, que rompe con la cohesión dentro de la familia real saudí, por la crisis económica, que amenaza con las promesas de modernización y estabilidad económica y por sus constantes represiones a toda oposición, que cuestionan su supuesta imagen reformista.

Palabras clave: Arabia Saudita; autoritarismo; religión; modernización; conservadurismo.

\footnotetext{
* Artículo recibido: 19 de febrero de 2021 / Aceptado: 3 de agosto de 2021 / Modificado: 11 de agosto de 2021. Este artículo es producto de la tesis de grado "Reformismo saudí ¿nuevas bases estructurales?" presentado en la Universidad de Congreso, Facultad de Estudios Internacionales, Argentina. Dicho trabajo fue reconocido por las autoridades de la Universidad. La presente investigación no contó con financiación.

** Licenciado en Relaciones Internacionales por la Universidad de Congreso, Argentina. Profesor y tutor de la Licenciatura en Relaciones Internacionales, Universidad de Congreso, Facultad de Estudios Internacionales. Investigador del continente europeo en el Observatorio Universitario de Terrorismo (OUT) del Instituto de Relaciones Internacionales, Argentina. Correo electrónico: jose.e9712@gmail.com iD https://orcid.org/0000-0003-2988-4471
}

\section{Cómo citar}

Teruel, J. (2022). Mohammed bin Salmán: legitimidad y nuevos desafíos. FORUM. Revista Departamento Ciencia Política, 21, 254-275. https://doi.org/10.15446/frdcp.n21.93768 


\title{
Mohammed bin Salman: Legitimacy and New Challenges
}

\begin{abstract}
This article aims to analyze the legitimacy of the Crown Prince of Saudi Arabia, Mohammed bin Salman, considering the historical pillars of legitimization of the crown and its current situation. The research is addressed through a qualitative approach while the hypothetical-deductive method is used by formulating a guiding question and establishing from it the objectives and hypothesis of the investigation, which will be tested by using rational reflection and observation of reality, through a documentary analysis of printed and digital books, press releases, publications in electronic journals and government reports. The hypothesis is that Mohammed bin Salman's legitimacy is weakened by his growing personalism, which breaks with cohesion within the Saudi royal family, due to the economic crisis, which threatens the promises of modernization and economic stability and because of his repression of opponents, which questions his alleged reformist image.
\end{abstract}

Keywords: Saudi Arabia; authoritarianism; religion; modernization; conservatism.

\section{Introducción}

Arabia Saudita ha sido un enigma para muchos académicos de las relaciones internacionales. El autoritarismo de la monarquía es la principal causa del hermetismo del reino, por el cual no es sencillo obtener una mirada crítica de lo que sucede en su interior. No obstante, las declaraciones de la monarquía durante los últimos años sugieren que Riad está buscando un cambio de rumbo por medio de reformas de carácter económico, político y social y, a pesar de que algunas de ellas se realizaron a principios del presente siglo, no se habló realmente de un "reformismo" hasta que el príncipe heredero, Mohammed bin Salman, presentó en 2016 su ambicioso plan modernizador llamado Visión 2030.

Sin embargo, el carácter reformista del príncipe heredero está siendo cuestionado por los sectores progresistas y grupos de derechos humanos alrededor del mundo, especialmente desde 2018, cuando el periodista Yamal Jashogyi fue asesinado en un consulado de Arabia Saudita en Estambul (Turquía) y todas las miradas apuntaron al hijo del rey Salman, quien hoy es la mayor figura política del país. Desde entonces, Mohammed bin Salman ha intentado, aunque sin éxito, limpiar su imagen. La cumbre del G20 en Riad era una de sus oportunidades para rehabilitar su imagen y mostrarle al mundo las reformas que se están llevando a cabo en el reino, pero la pandemia del coronavirus frustró sus planes. 
Por otra parte, la ruptura de la tradición de la toma de decisiones por medio de mecanismos de consenso y consulta en pos de un modelo personalista por el cual se centraliza el poder en la figura de Mohammed bin Salman, ha provocado que los miembros de la familia real hoy estén enfurecidos con el hijo del rey (Hernández Martínez, 2020). Como si ello fuera poco, la crisis económica producida por la caída de los precios del petróleo, dificulta los planes modernizadores de Mohammed bin Salman y debilita uno de los pilares históricos de legitimidad para la corona, la distribución de las riquezas petroleras entre los miembros de la población.

El heredero al trono tiene un pequeño margen de acción. Su dinámica de reforma y represión busca, por un lado, postularlo como el representante del futuro de Arabia Saudita; y por el otro lado, silenciar las voces que buscan impedir que esta visión se concrete o que ponen en duda su carácter reformista. Esto se complementa con un discurso nacional populista con culto a su persona, que le permite acusar de traidor de la nación a todo aquel que se oponga a su gobierno, justificando así sus represiones y culpar a enemigos internos y externos de sus propios fracasos para evitar las críticas en su contra.

\section{Metodología}

La investigación es abordada desde el enfoque cualitativo. Respecto del método, se utiliza el hipotético-deductivo, buscando combinar la reflexión racional con la observación de la realidad para comprobar la hipótesis propuesta. En cuanto a las técnicas, se realiza la investigación a partir del análisis documental por medio de libros impresos y digitales, notas periodísticas, publicaciones en revistas electrónicas e informes gubernamentales.

La pregunta que guía la investigación es cómo ha afectado a la legitimidad del príncipe heredero saudí su creciente personalismo, la crisis económica que afronta el reino y las constantes represiones. Esta fue formulada con el objetivo de analizar la legitimidad del príncipe heredero de Arabia Saudita, Mohammed bin Salman, considerando los pilares históricos de legitimación de la corona y su situación actual. De manera específica, se busca determinar los pilares de legitimidad de la Casa Saud y su impacto en los procesos históricos saudíes, identificar las clases sociales saudíes y el lugar que ocupan en la estructura social y analizar la legitimidad del príncipe heredero a través de sus políticas y su impacto sobre los pilares legitimadores de la monarquía saudí.

La hipótesis de la investigación es que la legitimidad de Mohammed bin Salman se encuentra debilitada a causa de su creciente personalismo, que rompe con la cohesión dentro de la familia real saudí, por la crisis económica, que amenaza con las promesas de modernización y estabilidad económica y por sus constantes represiones a toda oposición, que cuestionan su supuesta imagen reformista. 
Con el fin de comprobar esta hipótesis, se realiza, en primer lugar, un estudio de las bases sobre las cuales se funda Arabia Saudita y sobre su desarrollo a través del tiempo, tomando como punto de partida el Primer Estado Saudí, también conocido como el Emirato de Diriyah, fundado en 1744 por el Pacto de Diriyah entre el emir Mohammed bin Saud y el imam Mohammed bin Abdul Wahab. Esto permite entender el papel de la corriente wahabita como fuente de legitimación en Arabia Saudita y también ayuda a advertir las causas de las crisis políticas más importantes que ha sufrido el reino en el pasado. Luego se hace un estudio de la estructura social y de poder en la Arabia Saudita actual, con el fin de detectar los principales pilares de legitimación y la relación que existe actualmente entre la corona con las distintas clases sociales. Posteriormente, se analiza la situación actual del reino y se desarrollan las causas de la debilitación de la legitimidad del príncipe heredero. En suma y reflexionando sobre los argumentos expuestos, se hacen las consideraciones finales.

\section{La legitimación desde el Primer Estado Saudí y los intentos modernizadores}

Para entender las bases sobre las que se funda Arabia Saudita y cómo se ha legitimado históricamente la corona del reino, debemos remontarnos a sus inicios, en 1744, cuando se acordó el Pacto de Diriyah entre el emir Mohammed bin Saud y el imam Mohammed bin Abdul Wahab, con la intención de crear un Estado uniendo las tribus de Arabia y de propagar la doctrina wahabí para legitimar sus conquistas. En aquel momento, bin Saud necesitaba de un respaldo religioso para preservar un sistema de impuestos que iban en contra de la sharía o ley islámica, mientras que bin Abdul Wahab necesitaba de fuerzas militares para expandir su visión conservadora del islam. Mohammed bin Saud le prometió el apoyo militar a cambio de dos condiciones: que se quedara en Diriyah y que no se opusiera al sistema de impuestos. Mohammed bin Abdul Wahab aceptó la primera condición, pero no la segunda, asegurando que el botín de guerra conseguido por su causa sería mucho mayor que el de los impuestos. Acabar con estos impuestos le dio mayor popularidad a bin Abdul Wahab en Diriyah. El emir le juró al clérigo protección y lealtad ante su visión del islam, siendo la base para los Estados saudíes (Wynbrandt, 2010, p. 117).

1. El wahabismo es una corriente reformista islámica del tipo tradicionalista neohanbalí. Este propone devolver al islam a su pureza, la erradicación chií y la aplicación de la ley islámica en la comunidad, la cual es guiada por un imam, quien tiene el deber de los principios y las prácticas religiosas (Waardenburg, 2019). 
El Emirato de Diriyah, también conocido como el Primer Estado Saudí logró expandirse hasta los límites del Imperio Otomano, en al-Hasa por el este e Hiyaz por el oeste, convirtiendo a los Saud en una amenaza. Por esta razón, en agosto de 1811, el gobernador de Egipto, Mohammed Alí, quien administraba en nombre del Imperio Otomano, emprende por encargo del sultán, Mahmut II, una campaña contra los wahabíes buscando recuperar Hiyaz, restableciendo para 1815 la autoridad otomana en Medina y La Meca, por lo que los Saud se vieron obligados a retirarse a Neyd. Ese mismo año, Alí deja a su hijo, Tusan, a cargo de la campaña contra los wahabíes y él se encarga de gobernar Egipto. Un año más tarde, Tusan muere y es sucedido por su hermano Ibrahim, quien inició la campaña de conquista de Neyd, utilizando el apoyo de tribus beduinas para lograr su objetivo. Para 1818 los egipcios llegan a Diriyah, la capital del emirato homónimo y logran la rendición del emir Abdullah bin Saud, finalizando la guerra Otomana-Wahabí con la desaparición del Emirato de Diriyah. Para evitar que Neyd supusiera un riesgo para los otomanos, Ibrahim ordenó la deportación de miembros de la familia Saud y de los descendientes de Mohammed bin Abdul Wahab a Egipto (Cuesta, 2018).

Solo seis años después, Turki bin Abdullah, nieto de Mohammed bin Saud, expulsaría a los egipcios de Riad y sus alrededores, dándole comienzo al Segundo Estado Saudí, el Emirato de Neyd. Una de sus primeras acciones de Turki fue lograr que las familias que habían huido a Egipto volvieran para luchar por la reconstrucción del emirato. No obstante, es necesario aclarar que este emirato tuvo grandes diferencias con los otros Estados saudíes. En primer lugar, no se logró controlar ni La Meca ni Medina, dos ciudades sagradas para el islam, por lo que se considera a este emirato como el más desconectado con la religión y más frágil en legitimidad. En segundo lugar, las debilidades internas eran muy grandes y fue esto lo que lo condujo a su caída en 1891. Tras el asesinato de Turki en manos de su primo, Mishari bin Abdul Rahman y de que este tomara el poder, el hijo de Turki, Faisal bin Turki, quien se encontraba en una operación militar en al-Hasa, regresó a Riad con sus tropas y mató a Mishari, quedando al mando del emirato. Mientras se desarrollaban estas luchas internas, Mohammed Alí se había rebelado contra el poder otomano y había conquistado Siria. En su proyecto de reino independiente, Alí planeó la conquista de Hiyaz y el establecimiento de un gobierno títere en Neyd. Faisal no pudo impedir la invasión egipcia en Neyd y se retiró a al-Hasa. En su lugar, Alí estableció a Khalid bin Saud, hermano de Abdullah bin Saud al mando. En 1838 Faisal fue capturado por los egipcios y enviado a El Cairo, sin embargo, el rechazo de Austria, Prusia, Rusia y especialmente de Gran Bretaña a la emergencia de un poder ajeno al Imperio Otomano en la región, obligó a Alí a desistir de sus planes expansionistas y, tan pronto como las fuerzas egipcias abandonaron Neyd, el poder de 
Khalid se debilitó, siendo expulsado de Riad en 1841 por Abdullah bin Thunayan, bisnieto de Thunayan bin Saud, quien era hermano del fundador del Primer Estado Saudí, Mohammed bin Saud.

Para 1843 Faisal escapó de El Cairo y retomó el poder del emirato, luego de que Abdullah muriera bajo circunstancias poco claras, teniendo que enfrentar Faisal disidencias de los emires en la provincia de Qasim. Tras la muerte de Faisal en 1865 comenzó una larga lucha por el trono entre sus hijos Saud, Abdullah y Abdul Rahman que duraría décadas. En primera instancia la pelea fue entre Abdullah y Saud. El primero fue quien sucedió a Faisal bajo el principio de primogenitura, sin embargo, Saud reclamaba el trono bajo el argumento del poder que le había conferido su padre en vida al legitimarlo para gobernar Neyd meridional.

En 1871 Saud depuso a Abdullah y se hizo con el poder, aunque sería depuesto en el mismo año ya que Abdullah pidió ayuda al gobernador otomano en Bagdad, Midhat Bajá, quien no solo lo reestableció en el trono, sino que aprovechó la situación para tomar al-Hasa. La división también se manifestaba entre los ulemas, ya que algunos creían que Abdullah era legítimo heredero, mientras que, para otros, como él en un intento por eliminar la oposición de Saud había solicitado ayuda de los otomanos, estaba desautorizado para gobernar. Saud recuperó el mando en 1873 con el apoyo de los grupos tribales ajmanitas y dawasires (Crawford, 1982). Sin embargo, ante la muerte de Saud en 1875, comenzó una pelea entre sus dos hermanos, Abdullah y Abdul Rahman. En esta ocasión los ulemas wahabíes le mostraron lealtad a Abdul Rahman, quien comenzó a gobernar el emirato.

No obstante, en 1876 Abdullah regresó del exilio reclamando el trono y Abdul Rahman fue presionado para abdicar y aliarse con su hermano contra los intereses de los hijos de Saud, quienes también reclamaban el trono. En 1887 los hijos de Saud depusieron a Abdullah, quien pidió ayuda al gobernador de Hail, Mohammed bin Rashid. Él aprovechó las disputas familiares para apoderarse de Riad, expulsando a los hijos de Saud y colocando a uno de sus comandantes de confianza, Salim al-Sibhan como gobernador del territorio adquirido. Para 1879 Abdul Rahman regresó a Riad y ejerció el poder como vasallo de al-Sibhan, aunque no estaba dispuesto a ser un gobernante títere, por lo que buscó el apoyo de los gobernantes de Qasim. Qasimíes y rashidíes lucharon en la batalla de Mulaida en 1891, triunfando estos últimos. Abdul Rahman huyó a Kuwait donde fue acogido por los al-Sabah, quienes serían determinantes en el siglo XX para que los Saud recuperaran su poder (Cuesta, 2018).

Transcurrió una década hasta que Abdulaziz bin Saud, hijo de Abdul Rahman, creó una milicia religiosa llamada Ijwan² luchó contra los Rashid, los expulsó de Riad y comenzó

2. La traducción del árabe sería: "Hermanos". 
a expandirse a Qatif, Yubail y al-Hasa. Este brazo armado de monjes soldados pretendía dividir a la población beduina, establecerla alrededor de los oasis junto con los otros habitantes y transformarlos de asaltantes a soldados del islam. Sus técnicas se basaban en matar a quienes se opusieran, profanar lugares santos de las diferentes tribus y obligarlos a convertirse. Los Ijwan fueron extremadamente efectivos hasta que, en 1924 luego de la toma de Hiyaz y por ende de La Meca, Medina y Yeda, dejaron de ser útiles para la corona, la cual quiso terminar con el expansionismo y crear un Estado moderno. Ante esta situación, los Ijwan se volvieron en contra del rey, considerando como un pecado los fuertes lazos de la dinastía con los británicos, quienes les enviaban armas y dinero, lo que los llevó a protagonizar la batalla de Sabilla en 1929, en la cual bin Saud triunfó y este brazo armado dejó de existir (Silverfarb, 1982).

Tres años más tarde, en 1932, se creó oficialmente Arabia Saudita bajo un sistema monárquico absolutista legitimado por medio de la doctrina wahabita. El papel del islam es central, lo que se ve reflejado en diferentes aspectos. En primer lugar, los símbolos religiosos se encuentran presentes incluso en la misma bandera del reino, la cual es de color verde -color sagrado del islam-y tiene escrita la shahada o profesión de fe, cuya traducción textual es "No hay más Dios que Alá y Mahoma es su profeta".

Por su parte, el marco institucional también está caracterizado por una fuerte presencia islámica. La mayor autoridad religiosa es el gran muftí, nombrado directamente por el rey y es quien preside el Comité Permanente de Investigación Islámica y Emisión de fetuas - decisión que da el muftí a una cuestión jurídica-, teniendo como tarea principal dar su opinión sobre asuntos legales y sociales del reino. Por otro lado, en el territorio continental, la aplicación de la sharía está articulada por el máximo organismo religioso nacional, el Consejo de Expertos Superiores Religiosos, mientras la mutawa o policía religiosa se encarga de que estas normas se respeten en el ámbito civil. Sin dudas, el elemento de mayor legitimación del monarca es específicamente una de sus funciones, la de "Guardián de los Santos Lugares", teniendo como tarea proteger las dos ciudades más santas en el islam: La Meca y Medina (Baamir, 2016, p. 29).

La rama wahabita tiene peso ideológico considerable para el desarrollo político. El oficialismo religioso se encarga del control de la cultura, la justicia y la educación, influyendo sobre la percepción que tienen los ciudadanos sobre la política. A cambio de la protección de la realeza y de dichos poderes, los estamentos wahabitas aseguran presentar ante las comunidades musulmanas un discurso congruente con la autoridad saudí (Hernández Martínez, 2020).

Sin embargo, el papel central que tuvo esta corriente religiosa conservadora desde los inicios del Estado saudí ha dificultado históricamente la modernización del reino, por lo que los monarcas han tenido que idear formas de evitar que la población se rebelara 
en estos procesos, aunque no siempre estas políticas fueron exitosas y, en muchos casos, Riad tuvo que dar pasos atrás en sus procesos modernizadores, incluso viéndose obligados a dictar políticas más conservadoras que las que existían antes de las reformas.

Retomando la historia del reino, un año después de su creación, en 1933, bin Saud firmó una concesión de exploración petrolera con la empresa Standard Oil, sin embargo, no sería hasta 1938 que encontrarían petróleo. La Arabia Saudita prepetrolera era burocráticamente débil y debía gran parte de sus ingresos a las peregrinaciones de los fieles, a las aduanas, a los impuestos cobrados por la mutawa y a subsidios británicos. La decadencia del poder británico fue aprovechada por Estados Unidos. Durante el paso de los años, la filial de la petrolera norteamericana, que para 1944 pasaría a llamarse Saudi Aramco, se convirtió en un motor de modernización, construyendo aeropuertos, redes ferroviarias, hospitales, buscando reservas subterráneas de agua potable e investigando sobre nuevas posibilidades agrarias (Harrigan, 2016, p. 79).

En 1953 el rey bin Saud murió, asumiendo su hijo Saud bin Abdulaziz, aunque no sin antes sufrir una disputa con su hermano Faisal, quien consideraba que era incompetente para el cargo. Si bien Saud bin Abdulaziz tuvo acciones destacables como la fundación de la primera universidad del reino, llamada Universidad del Rey Saud, la participación en la creación de la Organización de Países Exportadores de Petróleo (OPEP) o la creación del Ministerio de Petróleo y Minerales, también fue criticado por el restablecimiento de la esclavitud, el incremento de la deuda y la supresión del cargo de primer ministro, centralizando el poder en su persona (Harrigan, 2016, p. 91). Incluso los hermanastros del rey, empezaron a temer que elegiría a uno de sus hijos para sucederle, ya que el gobernante los designó en puestos de poder: Saud Fahd en el Ministerio de Defensa, a Musaid en la Guardia Real, a Khalid en la Guardia Nacional y a Saad en la Guardia Especial (Al-Rasheed, 2010, p. 114).

En 1962 luego de que el rey saliera del país por problemas médicos, su hermano, Faisal propondría un plan de gobierno de 10 puntos, entre las que se destacaban la creación de un consejo judicial y la abolición de la esclavitud. A su vuelta, Saud amenazó con movilizar a la Guardia Revolucionaria, aunque ellas acabaron por levantarse contra él y los ulemas apoyaron a Faisal, por lo que en 1964 se vio obligado a abdicar y exiliarse en Ginebra, dejando a su hermano en el poder (Al-Rasheed, 2010, p. 117).

Faisal pretendió realizar desde el comienzo una modernización en el sector público saudí, caracterizado por la falta de instituciones y la debilidad de las estructuras burocráticas. Así, creó departamentos y organizaciones gubernamentales, incluyendo un poder judicial, escuelas y universidades y envió a estudiantes al extranjero, quienes más tarde se convertirían en la piedra central de la administración saudí (Rich, 2018). 
El monarca entendía que una modernización repentina podría generar que la población se rebelara. Por ello, no solo otorgó subsidios a organizaciones tribales, sino que también creó un plan de dos pilares. El primero era la legitimación por medio de la religión y el segundo era estrechar relaciones con Estados Unidos, con quienes tenía intereses comunes en la lucha contra el comunismo y en el desarrollo de la industria petrolera, la cual sería fundamental para modernizar la economía (Bosemberg, 1998).

Arabia Saudita comenzó a dar aportes a otros países de la región para el establecimiento de diarios, mezquitas y fundaciones, siempre teniendo en cuenta el grado de antisovietismo y reislamización. Así, el reino buscaría también el desarrollo de los programas educativos religiosos, convirtiéndose en el bastión sunita. La exportación del wahabismo sería fundamental para la lucha contra el panarabismo, sobre todo representado por la Federación de Repúblicas Árabes (Bosemberg, 1998).

En 1970 llegaría el "Primer Plan Quinquenal” (1970-1975) que consistía en diversificar la economía para reducir la dependencia del crudo. Con este fin se crearon empresas petroquímicas, de hierro, cemento, acero y fertilizantes, como también comenzaron las grandes compras de tecnología y armamento. Al cabo de 5 años, se veían avances importantes en el reino, con la aparición de una clase media y de una clase obrera y con la mejora de la calidad de vida. Para detener cualquier oposición o creación de sindicatos, el rey Faisal realizó un pacto en el que evitaba estos tipos de organizaciones y a cambio aportaba salud y educación gratuita (Bosemberg, 1998).

El plan quinquenal acabó el mismo año en que el rey fue asesinado por su sobrino, Faisal bin Musaid, en venganza de su padre y de su hermano, fue asesinado en una represión durante una manifestación en 1965 (Cuesta, 2020). La línea de sucesión llevó a Khalid bin Abdulaziz a ser el siguiente monarca. Recién asumió su cargo, estableció el "Segundo Plan Quinquenal" (1975-1980) que era muy diferente al primero. En este se planteaba la maximización de recursos mineros y petroleros por medio de la especialización en el desarrollo de recursos, se comenzó a restringir la oferta petrolera para elevar los precios y se establecieron empresas nacionales en países limítrofes, con el fin de satisfacer demandas nacionales y exportar excedentes a Estados Unidos, Europa y Japón (Nehme, 1994).

De esta forma, la sociedad saudí logró grandes avances: las estructuras tribales se debilitaron ya que muchos miembros se convirtieron en obreros y sus líderes se enriquecieron; grupos nómadas se volvieron sedentarios gracias a las oportunidades laborales y a las parcelas a títulos individuales que se otorgaron y un sistema clientelista mantuvo a la población controlada (Bosemberg, 1998). Sin embargo, todo cambiaría en 1979 con la toma de la Gran Mezquita de La Meca. Esta revuelta fue planeada por un 
grupo de tradicionalistas religiosos que criticaba una desvinculación entre el wahabismo fomentado por las mezquitas, el sistema educativo y los medios de comunicación, así como las reformas impulsadas por Riad.

Si bien la corona decidió acabar la revuelta por la fuerza, con ayuda francesa y la luz verde de los ulemas, matando a miles de personas, usando armas químicas y luego decapitando a los organizadores del motín, esto fue un aviso de que había sectores disconformes con las modernizaciones y de que podría ser el primero de muchos levantamientos (Kechichian, 1990). Por ello, las políticas futuras se basaron en un retorno al conservadurismo, ahora incluso más estricto que antes: las mujeres fueron obligadas a usar la abaya, se les prohibió aparecer en televisión y en la prensa, le otorgaron más poder a la policía religiosa, se cerraron los cines y las tiendas de música y se aplicó la segregación de sexos. Además, ese mismo año triunfaba la Revolución Islámica en Irán, derrocando al shah e instalando un sistema híbrido en el país, combinando rasgos de una teocracia como otros propios de la democracia. Desde entonces, la lógica regional sufriría cambios. Los saudíes exportarían el wahabismo, pero ahora para luchar contra la influencia chií, exteriorizada por los persas (Bosemberg, 1998).

La guerra del Golfo traería nuevos problemas para la monarquía saudí. La presencia extranjera en la región y, sobre todo, la estadounidense en el norte de Arabia Saudita generaría críticas de los sectores más conservadores, molestos no solo por ver estas tropas en el territorio, sino también porque Riad no podía establecer un orden regional a pesar de los altos gastos militares (Galindo, 2006, p. 342). Además, la crisis del petróleo y el posterior desarrollo de nuevas fuentes energéticas en el mundo provocaron que el reino entrara en una crisis por la dependencia económica con el crudo. En este contexto, para calmar a la población, Riad tuvo que hacer reformas sociales y económicas. Se quitaron subsidios, aumentaron los servicios, se privatizaron empresas, se creó la $S_{\text {Shura }}{ }^{3}$, se censuraron programaciones televisivas extranjeras, se profundizó la educación religiosa y se prohibió la enseñanza de "doctrinas occidentales" (Bosemberg, 1998).

El siglo XXI supondría nuevos desafíos para el régimen saudí. Los atentados del 11 de septiembre de 2001 ejecutado por Al Qaeda, bajo el mando del saudí Osama bin Laden y en el que participaron 19 terroristas, de los cuales 15 eran ciudadanos saudíes, significó un desafío para la alianza entre Estados Unidos y Arabia Saudita. Las investigaciones develaron que los jóvenes terroristas no eran fanáticos sin educación o de grupos excluidos, sino que pertenecían a la clase media y estaban bien educados, lo que pone el acento en la desocupación de los menores de 25 años en el reino.

3. Consejo consultivo de la corona. 
Rápidamente Arabia Saudita condenó el ataque y envió condolencias al presidente estadounidense George W. Bush. Días después, el 20 de septiembre el ministro saudí de Asuntos Exteriores le aseguró a Bush la completa cooperación en la lucha contra el terrorismo, sin embargo, en Estados Unidos el hecho de que la mayoría de los terroristas fueran saudíes fue visto como una actitud de antinorteamericanismo. El ataque produjo un gran antagonismo entre las dos naciones: a Arabia Saudita se la culpó por la exportación de la doctrina wahabita y por financiar a grupos que apoyaban el terrorismo, mientras que Estados Unidos fue culpado por su incondicional apoyo a Israel, lo que fue presumido como una de las causas del ataque (Wynbrandt, 2010, p. 277). No obstante, la inestabilidad en la región y la necesidad norteamericana de un aliado para contrarrestar la amenaza iraní con sus tentativas de proceder con su política de enriquecimiento de uranio actuaron en un sentido contrario, denotando la importancia de Arabia Saudita en la política exterior estadounidense.

En el interior del reino, las demandas sociales de modernización en el siglo XXI, entre las que podemos destacar la petición de 2003 "en defensa de la nación" que culpaba a los islamistas y conservadores por la inestabilidad nacional, contribuyeron en los procesos reformistas siguientes (Galindo, 2006, p. 352). Abdullah bin Abdulaziz, medio hermano de Fahd, asumió en el año 2005, aunque gobernó de facto desde 1995 puesto que su predecesor estaba incapacitado por una hemiplejía que le impedía mover parte de su cuerpo. El monarca encaró distintas modernizaciones en las que participó su hermano, sucesor al trono y actual rey Salman bin Abdulaziz. Cabe aclarar que durante el reinado de Abdullah las reformas se hicieron a un ritmo lento por las resistencias internas y dificultades derivadas de la primavera árabe (Hernández Martínez, 2020).

Algunos de los mayores logros del rey fueron garantizar en 2011 el derecho al voto femenino y las candidaturas de mujeres a las elecciones municipales previstas para 2015, la creación de la Universidad de Ciencia y Tecnología rey Abdullah, así como permitir que hombres y mujeres pudieran compartir ciertos espacios. Desde el 2015, el rey es Salman bin Abdulaziz. Él se propuso hacer cambios que resultaron fundamentales: decidió que el heredero al trono no fuera su sobrino, Mohammed bin Naif bin Abdulaziz y, en cambio, propuso al hijo, Mohammed bin Salman, quien fue aceptado por la Comisión de Lealtad. La elección de Mohammed, un joven de 32 años, corresponde con que dos tercios de los saudíes son menores de 30 años y que su pensamiento modernizador era popular entre las nuevas generaciones. Hoy él es el epicentro del poder saudí. 


\section{Estructura social y distribución del poder en Arabia Saudita}

Arabia Saudita es una monarquía absolutista en la cual, si bien la política está intrínsecamente ligada a las decisiones del rey, las dinámicas sociales y de poder se encuentran condicionadas por la correlación de fuerzas entre los diferentes actores y grupos. La sociedad saudí no solo queda caracterizada por condicionantes económicos, sino que también por elementos políticos y religiosos.

Actualmente, en la sociedad saudí, compuesta por 34 millones de personas, se distinguen siete clases diferentes (Hernández Martínez, 2020). En la cúspide de la pirámide social se encuentran los príncipes saudíes y el oficialismo wahabita, herencia misma del Pacto que sirvió como base del Primer Estado saudí. La lógica es la siguiente: mientras que el clan familiar se compromete a proteger la corriente religiosa de críticos y difundir su interpretación del islam, los estamentos wahabitas presentan ante la comunidad un discurso coherente con el de la autoridad real.

En el país es una costumbre que el rey se deje asesorar por sus familiares más cercanos y por las figuras religiosas más relevantes del país. El reparto de tareas y la participación de los príncipes en el poder han asegurado históricamente la cohesión dinástica y la prevalencia del modelo. Romper con este factor podría romper con la estabilidad del régimen.

En el segundo escalón se encuentra una clase privilegiada y cercana a la familia real y el wahabismo. Esta clase privilegiada está formada por todos aquellos clanes y tribus que desde el momento de la reunificación se mantuvieron leales a la corona saudí. Este grupo también está constituido por aquellas personas que pudieron progresar a través de la administración pública y de las principales empresas nacionales.

El tercer estamento de la pirámide social está compuesto por trabajadores occidentales y de los países árabes del entorno, que representan a las corporaciones y delegaciones políticas con intereses económicos en el país. Se estima que el número de trabajadores extranjeros ronda los 11 millones de personas, comprendiendo así el 70 \% de la fuerza laboral.

En el cuarto lugar se encuentran aquellos saudíes que constituyen el prototipo de ciudadano promedio. No poseen un estatus especial ni son cercanos a las elites nacionales, pero el Estado les garantiza cierta calidad de vida y mínimos servicios de educación, sanidad y posibilidades laborales. El clientelismo favorece la dependencia económica de la mayor parte de los ciudadanos y reduce las posibilidades de que se creen movimientos contestatarios.

En el quinto escalón se ubican los ciudadanos saudíes críticos del gobierno, cuyas movilizaciones y reivindicaciones no son toleradas por el Estado. Los opositores son reprimidos o condenados, o bien deben huir del país y son señalados como elementos peligrosos para la estabilidad saudí. 
En el sexto peldaño se encuentran los saudíes que viven en la pobreza, siendo las clases más bajas del país. Se estima que unas cuatro millones de personas viven en la miseria, dependiendo de la solidaridad de congregaciones religiosas y algunas ayudas públicas. Son un sector de la sociedad invisibilizada y cuyas demandas son escasamente atendidas por las autoridades.

La base de la pirámide social está compuesta por la mano de obra extranjera encargada de los trabajos de poca cualificación. Gran parte de ellos provienen de países de Asia-Pacífico y de África y el régimen laboral al que están sometidos es duro y con escaso reconocimiento legal.

Por su parte, el limitado margen de participación político y social en Arabia Saudita genera que la sociedad quede marcada entre los partidarios del régimen y sus críticos. La decisión de la mayoría de los ciudadanos es la de mantenerse bajo los cánones políticos impuestos por la monarquía, ya sea por convencimiento ideológico, por miedo a la represión o, la tendencia mayoritaria, por indiferencia política.

Se puede mencionar, además, que existen dos pilares clave de legitimación de la corona, claramente visibles en esta estructura social. El primero de ellos es el wahabismo, el cual tiene una alta presencia institucional en el reino, pero también un peso ideológico para el desarrollo político. Esta corriente religiosa está fuertemente presente en la sociedad e influye en las apreciaciones de la sociedad y en su relación con el poder. El segundo pilar, es la distribución de la riqueza petrolera en determinados sectores sociales. Esto, como vimos en el estudio de la historia saudí, ha creado un lazo de dependencia de esta denominada "clase media" y busca impedir las movilizaciones en contra de la corona. Sin estos dos pilares, la estabilidad saudí corre peligro (Galindo, 2006, p. 338).

\section{La legitimidad de Mohammed bin Salman y sus nuevos desafíos}

Como se mencionó, Mohammed bin Salman entró en la escena principal de la política saudí con una imagen de reformista. En 2016, el actual príncipe heredero saudí, presentó el plan Visión 2030, un proyecto modernizador que refleja las metas y expectativas para tal año y que en Riad aseguran que es alcanzable ya que tiene en cuenta las fortalezas y debilidades del reino. Las principales metas de Visión 2030 son diversificar la economía, reducir la dependencia de los hidrocarburos y desarrollar sectores públicos como la salud, educación, infraestructura y el turismo. Es en este contexto que se han privatizado empresas estatales, se sacó a la bolsa a la petrolera Aramco y se abrieron islas para el turismo.

Si bien los objetivos principales son económicos, ellos significan un inevitable impacto en la sociedad. Así, la sociedad saudí pareciera estarse transformando de forma 
progresiva: se habilitaron los cines y teatros; las mujeres ahora pueden manejar, practicar deportes, presenciar espectáculos deportivos, ser parte de las fuerzas armadas, transitar sin una abaya ${ }^{4}$, abrir su propio negocio e incluso ya la policía religiosa no puede detener ni golpear a la gente con sus largos bastones.

El discurso del príncipe heredero es nacional populista basado en un culto a su persona. De manera constante recuerda a la audiencia de su juventud y su presunto pensamiento modernizador, presentándose como el modelo que deben seguir los saudíes si pretenden ser considerados como una nación moderna. Su juventud se muestra en su actividad en las redes sociales, especialmente en Twitter, así como en su aparición ante las cámaras bajándose de autos de carreras o acudiendo a conciertos. El nuevo plan nacional involucra proyectar la nueva nación como joven, moderna y con equidad de género.

El nuevo ciudadano no es más aquel beduino que criaba camellos ni el devoto que recibe beneficios de bienestar, sino que se espera que sea un sabio de la economía, un emprendedor arriesgado, un consumidor de una gran gama de productos y un pionero de los servicios neoliberales. Como el príncipe heredero, el saudí ideal se encuentra conectado al mundo por medio de las nuevas tecnologías. No obstante, los jóvenes necesitan más que nuevos espacios culturales y de entretenimiento como conciertos, partidos de fútbol o exhibiciones de arte, los cuales en realidad parecen ser herramientas de distracción de la situación interna. Existen demandas de suma importancia que deben ser atendidas, como más trabajos, menor inflación y, sobre todo, mayores libertades y respeto por los derechos humanos (Al-Rasheed, 2021, p. 170). Cabe decir que el discurso de bin Salman tiene un especial enfoque hacia la población menor de 25 años, que representa el $49 \%$ del total de los saudíes, pero ignora el otro $51 \%$.

Una de las narrativas nacionalistas dominantes es la de la protección de la nación del peligro del expansionismo iraní y sus proxies. Mohammed bin Salman frecuentemente es televisado visitando a soldados heridos en hospitales, demostrando la valentía de ellos y la voluntad de morir por la nación, mientras la media celebra el liderazgo del príncipe heredero y su generosidad al darles compensaciones. El discurso religioso también es utilizado en este contexto, sobre todo en el contexto de la guerra en Yemen. Los hutíes son representados por los medios como no musulmanes apoyados por Irán que no solo buscan la destrucción de Arabia Saudita, sino que también de La Meca. Los misiles interceptados dirigidos a Yeda, ciudad aledaña, sirve a la narrativa que el verdadero objetivo era atacar La Meca y que solo los paganos y no musulmanes podrían contemplar tal acto. 
Sin embargo, la función más importante de su discurso nacional populista es la justificación de las detenciones y ejecuciones para acallar a la gran variedad de críticos, dividiendo así al pueblo en dos grupos: los traidores - khains-y los verdaderos nacionalistas -watanis-. Los watanis son un grupo devoto a Mohammed bin Salman que no cree en la negociación ni en la persuasión, sino que frecuentemente hacen alusión al uso de la fuerza para eliminar enemigos internos y externos, a quienes culpan de todos los problemas que atraviesa el país, evitando cualquier tipo de evaluación de las políticas de la corona. Así, ellos no aceptan más que lealtad al príncipe heredero, por lo que acusan de traición, un crimen con pena de decapitación, a cualquiera que critique al régimen (Al-Rasheed, 2021, p. 172).

El nuevo nacionalismo también involucra escuadrones especiales entrenados y armados por Estados Unidos, encargado de silenciar las voces críticas del reino, como fue el caso de Yamal Jashogyi, de quien se hablará luego, armadas cibernéticas que intimidan y amenazan a activistas, tanto dentro del reino como en el exterior, rastrean cuentas disidentes y las hackean, utilizando tecnología china, estadounidense e israelí, entre otras. La persecución es una práctica común hacia los exiliados y presos de conciencia y sus familias, a quienes se los responsabiliza por las acciones de sus hijos, buscando crear una grieta entre ellos. Los familiares de los opositores son perseguidos y se les impide abandonar el país, como sucede con los padres de la activista por los derechos de la mujer Lujain al-Hatholoul (Al-Rasheed, 2021, p. 173).

El surgimiento de un discurso nacionalista basado en el concepto de una Arabia Saudita moderna excluye a todos aquellos que no se ajustan a él: las minorías religiosas, como los chíes y todos aquellos musulmanes cuya creencia no se adecue a la versión promovida por el reino, los musulmanes conservadores que se oponen al proyecto político "modernizador" y las regiones periféricas excluidas por el discurso de prosperidad de la nueva Arabia Saudita. La diversidad de la población, compuesta por diferentes tribus, grupos no tribales, inmigrantes, mayorías sunníes y minorías religiosas, viviendo en un territorio cuyas regiones han afrontado diferentes procesos históricos contribuye a que, lejos de homogeneizar, se provoque una fragmentación.

Desde hace años existe una campaña de represión contra los chiíes de Qatif, donde hubo grandes protestas en 2011 en el marco de la primavera árabe. Si bien la mayoría de los arrestados fueron liberados sin cargos, otros estuvieron bajo custodia sin cargos por uno o varios años, hasta que fueron juzgados por el Tribunal Penal Especializado, algunos de ellos afrontando la pena de muerte. Dos clérigos chí́es llamados Nimr al-Nimr y Tawfiq al-Amr pronunciaron sermones en 2011 que apoyaban la reivindicación de reformas políticas y religiosas. El primero fue ejecutado en el 2016 junto a otras decenas de personas, mientras que el segundo fue condenado a ocho años de cárcel. 
Como se mencionó anteriormente, el discurso nacionalista se utiliza para reprimir cualquier tipo de disidencia a la corona bajo la acusación de traición. No solo los periodistas y activistas son perseguidos, sino que todo aquel que se oponga al príncipe heredero, incluso su propia madre Fahda bint Falah al-Hathlin, fue puesta en prisión domiciliaria por órdenes suyas por oponerse a su nombramiento como sucesor al trono al creer que no estaba preparado (Carrión, 2018). Ni siquiera las redes sociales se encuentran libres del control gubernamental. En abril de 2021, Abdulrahman al-Sadhan trabajador en Riad de la Media Luna Roja de Arabia Saudita fue condenado a 20 años de prisión, por medio de un juicio secreto y sin representación letrada, bajo los cargos de defensa y financiamiento del terrorismo. Las pruebas que se utilizaron en su contra fueron tweets que criticaban el plan Visión 2030.

La ley antiterrorista sancionada en 2017 es un instrumento que utiliza frecuentemente la corona para reprimir a la oposición. Dicha normativa define al terrorismo como "la alteración al orden público". Definiciones tan amplias e imprecisas permiten que cualquier forma de activismo político sea enjuiciada como delito de terrorismo punible con largas penas de cárcel e incluso con pena de muerte (Josua, 2020). Además, esta ley le da el poder a la fiscalía pública para que detenga, arreste a las personas, intervenga sus comunicaciones y sus datos financieros e incaute sus bienes bajo sospecha de terrorismo y sin supervisión judicial. Si bien se creó un tribunal penal especializado para tratar los casos de terrorismo, este es en realidad funcional al régimen y solo institucionaliza las violaciones de derechos que ya existían anteriormente.

Empresarios, miembros de la familia real, activistas por los derechos humanos y feministas, periodistas, clérigos, académicos, minorías chíes, usuarios de redes sociales y familiares de exiliados son algunos de los grupos que aparecen entre las víctimas de las persecuciones gubernamentales. En noviembre de 2017 el príncipe heredero encerró a más de 200 personas en el hotel Ritz Carlton, entre las que se encontraban príncipes, empresarios, altos mandos militares y otros funcionarios, en el contexto de una "campaña anticorrupción", pero que buscaba contrarrestar a opositores a sus reformas y a la guerra en Yemen. Su primo Mohammed bin Nayef y su tío Ahmed bin Abdulaziz, dos personalidades que lo han criticado en múltiples ocasiones, también fueron detenidos años más tarde, en marzo de 2020.

El asesinato de Yamal Jashogyi incrementó el debate de la represión en el reino. Su asesinato se produjo en 2018 en un consulado de Arabia Saudita en Estambul, Turquía por un grupo saudí de intervención rápida formado por 15 agentes bajo las órdenes del príncipe heredero. El ex periodista del Washington Post que vivía en el exilio y que había trabajado para el gobierno durante el reinado de Abdullah bin Abdulaziz era sumamente crítico respecto de la represión de Arabia Saudita desde el ascenso de bin Salman, la 
cual consideraba insoportable. Es llamativo que constantemente omitía hablar de la represión antes de que el príncipe heredero se transformara en una de las figuras políticas más importantes del país. Jashogyi, luego de exiliarse, era criticado tanto por los defensores del reino, que lo consideraban un traidor, como también por grupos opositores, quienes sabían que él había trabajado para la monarquía. No obstante, poco a poco su postura fue siendo más crítica e incluso prometió crear puentes entre grupos opositores suníes y chiíes, activistas hombres y mujeres, jóvenes y adultos, lo que fue percibido como una amenaza por la corona, que decidió silenciarlo (Al-Rasheed, 2021, p. 137).

El informe de la Agencia Central de Inteligencia (CIA) de Estados Unidos publicado en febrero de 2021 y categóricamente rechazado por el gobierno saudí señaló al príncipe heredero como el responsable del asesinato de Jashogyi. Arabia Saudita admite que el opositor fue asesinado por sus agentes, pero niegan cualquier implicación de Mohammed bin Salman. Entretanto, el presidente estadounidense Joe Biden impuso sanciones a 76 ciudadanos saudíes acusados de amenazar a disidentes en el extranjero. Esta penalidad, llamada "prohibición Jashogyi" les impide entrar a territorio norteamericano. Sin embargo, cabe aclarar que el gobierno norteamericano tiene presente la importancia de Arabia Saudita para su política exterior en la región y por ello no sancionó al príncipe heredero.

Ciertamente, bin Salman es un reformista y un represor. Por un lado, se presenta como el símbolo de modernidad y del futuro próximo de la sociedad saudí, más tolerante y abierta, y por el otro mantiene vigentes políticas autoritarias. Presos de conciencia, juicios secretos y arbitrarios en los que los acusados no tienen representante letrado, represión de asociación y expresión pacífica, tortura, régimen de incomunicación a presos, ejecuciones extrajudiciales y crucifixiones ${ }^{5}$ son solo algunas de las violaciones que se reportan en el país.

El feminismo saudí es sin dudas uno de los aspectos que reafirman la dualidad de la imagen de bin Salman. Mientras el heredero al trono otorga derechos a las mujeres como manejar un auto o asistir a espectáculos deportivos, también considera al feminismo como un delito por implicar "ideas extremistas", con penas de flagelación y prisión. Por ello mismo, habría que preguntarse hasta qué punto los derechos otorgados no constituyen una mera "pantalla" para imponer una falsa idea de transformación. Asimismo, algunos de los avances si bien fueron presentados como modernizaciones sociales, tenían un trasfondo económico, tal es el derecho de emprender un negocio sin permiso de un tutor masculino.

5. Los sentenciados a tal práctica suelen ser degollados y luego expuestos en una cruz. Este es un método al que se condenó a chiíes partícipes de las protestas de 2011 y el objetivo es que, al exponer el cuerpo, la población tenga presente las consecuencias a las que se enfrenta si se opone a la corona. 
Son estas medidas autoritarias y la ausencia de reformas profundas las que causan que bin Salman no logre consolidar su apoyo por parte de los grupos más progresistas, quienes desconfían del supuesto carácter reformista del príncipe heredero. Y si bien académicos reconocidos como Abdullah Alaoudh ${ }^{6}$ afirman que gobernantes como bin Salman carecen de legitimidad popular ya que no son electos y que dependen del apoyo externo, esto no es realmente cierto (Harb, 2019). La estabilidad del reino depende, es verdad, del apoyo externo, pero también se ve condicionado por la situación dentro de la familia real y por la obediencia de los grandes sectores sociales.

El descontento también está presente en la Casa Saud y en el entorno de la corona, no solo producto de la purga de 2017, sino que principalmente porque bin Salman está rompiendo con la dinámica de consenso y consulta que había creado Abdulaziz bin Saud y que se constituyó como una tradición en el reino. En cambio, el príncipe heredero está imponiendo una forma de actuar más personalista y directa (Hernández Martínez, 2020). Antiguamente, los cargos de mayor relevancia solían repartirse entre los miembros de la familia real, pero ya no es así. Mohammed bin Salman ha tomado cargos estratégicos y ha designado a gente de su confianza que no forma parte de la Casa Saud en otras posiciones importantes: Ahmed Asiri asumió el cargo de subjefe de la Inteligencia General, pero fue despedido luego del asesinato de Yamal Jashogyi. Por su parte, Ahmed al-Khatib fue nombrado ministro de turismo, Turki al-Sheikh, antiguo ayudante del heredero al trono, fue ascendido a consejero real y encabezó primero la autoridad deportiva del reino y luego la autoridad de entretenimiento (Al-Kahmri, 2020).

La ruptura de la tradición ya había comenzado a notarse cuando el rey Salman le dio preferencia a su hijo no primogénito para sucederle en el trono, ahora se ve aún más con bin Salman y su acumulación de poder. Él no solo es príncipe heredero, también es ministro de defensa, dirige el Fondo Soberano de Arabia Saudita, es presidente del Consejo de Asuntos Económicos y de Desarrollo, presidente del Consejo de Asuntos Políticos y de Seguridad y presidente del Consejo Supremo de Saudi Aramco, entre otros. En otras palabras, tiene el control personal sobre la defensa, la economía y la política exterior del país.

Con ello, bin Salman busca ser el representante del futuro nacional y evitar cualquier oposición a sus planes. Sin embargo, existe otra gran amenaza para su legitimidad: la actual crisis que atraviesa el país y que, en consecuencia, pone en riesgo el cumplimiento de las metas propuestas en el plan Visión 2030. En efecto, el derrumbe de los precios del petróleo en 2020 profundizó un problema que ya venía desde el 2014, cuando

6. Investigador Principal del Centro para el Entendimiento Musulmán-Cristiano de la Universidad de Georgetown en Washington. 
los precios del barril sufrieron una fuerte caída, pasando de un pico de US\$̦ 105 en junio a menos de US\$ 60 en diciembre del mismo año (Daicz y Monlezún, 2016). Además, las limitaciones a la circulación impuestas en diversas ciudades han perjudicado el turismo y los planes de entretenimientos promovidos por el gobierno. La peregrinación a La Meca, una de las grandes fuentes de ingreso para los saudíes fue duramente afectada. La corona tuvo que impedir en el 2020 y 2021 el ingreso de musulmanes extranjeros y solo permitió a un número limitado para participar con distanciamiento social.

Ante este panorama, el gobierno saudí tuvo que aplicar medidas de austeridad en 2020 para paliar un déficit fiscal que ascendía a US \$ 9.000 millones en marzo. Así, la corona triplicó el Impuesto al Valor Agregado (IVA), pasando del 5 \% a un $15 \%$. Además, se eliminaron los subsidios a funcionarios estatales - medida que afectó a más de 2 millones de personas- y se detuvieron iniciativas y proyectos de reforma económica (Al-Rasheed, 2021, p. 332).

Cabe aclarar que no es la primera vez que el gobierno se ve obligado a tomar el camino de la austeridad. A fines de 2015 la corona ya había recortado bonificaciones, el pago de horas extras y otros privilegios que alguna vez fueron considerados beneficios de rutina del sector público saudí. En un país en el que, como se explicó anteriormente, la legitimidad depende en gran medida de la distribución de las riquezas petroleras entre los habitantes, la aceptación de las medidas de austeridad es crucial para que se mantenga la estabilidad de la realeza. De momento, el desempleo de $11.7 \%$ y la inflación cercana al 6 \%, impulsada por el aumento del IVA, son fuentes de malestar en la sociedad que ve incumplirse las promesas de bin Salman (Nereim, 2021). Aquí, el discurso de los enemigos internos y externos cobra gran importancia, culpando, por ejemplo, a los extranjeros por el desempleo.

\section{Conclusiones}

La imagen de Mohammed bin Salman es controvertida. El príncipe heredero se presentó en la escena nacional e internacional como un reformista, en un país gobernado por una monarquía absolutista que legítima su poder a través de dos pilares: el wahabismo, una corriente tradicionalista islámica que busca devolver al islam a su pureza perdida y que ha sido una de las bases sobre las que se construyeron los diferentes Estados saudíes, y la distribución social de las riquezas petroleras, que es propio del así llamado "Tercer Estado Saudí".

La emergencia del príncipe heredero vino acompañada por innovadores proyectos modernizadores enmarcados en Visión 2030, un plan que busca transformar la economía y la sociedad saudí para tal año y que refleja la visión que tiene Mohammed bin Salman respecto del futuro saudí. Reducir la dependencia de los hidrocarburos, diversificar la economía y desarrollar sectores como la educación, la salud y el turismo son algunas de sus metas. 
Que el plan puesto en marcha en 2016 represente la visión del heredero al trono sobre el futuro de Arabia Saudita implica, en primer lugar, que él constituye el epicentro de la política saudí y, también, que el futuro de Arabia Saudita está sujeto a él. Su discurso nacional populista con culto a su persona y sus políticas así lo confirman. En efecto, bin Salman constantemente busca que la media lo represente como un joven con pensamiento moderno y que resalte sus reformas, las cuales constituyen el camino hacia la Arabia Saudita del futuro, donde él es el rey. Esta cobertura le ha ayudó, al menos en un comienzo, a obtener apoyo internacional, indispensable para atraer inversores extranjeros y lograr que la liberalización de la economía y que la menor dependencia de la economía saudí para con el petróleo se concreten exitosamente.

No obstante, el creciente personalismo de bin Salman, marcado por la acumulación de poder por medio de la designación de sí mismo y de su gente de confianza en cargos de relevancia y de la exclusión de miembros de la familia real de ellos ha despertado rencores dentro de la Casa Saud. En efecto, mientras antiguamente existía una tradición de consulta y consenso entre el rey y los otros miembros de su familia, asegurando la cohesión y armonía, hoy es bin Salman quien controla la economía, la política y la defensa del país. El príncipe heredero ha demostrado estar dispuesto a purgar a todo aquel que le represente una amenaza. Esto sin dudas rompe con tal cohesión y genera oposiciones dentro de la misma familia real, siendo una amenaza para la estabilidad política saudí.

Por otra parte, el discurso que ha creado bin Salman en el que los saudíes están hechos a su imagen y semejanza y viven en un país moldeado con base en su visión de modernidad ha ayudado a justificar, en nombre de la nación saudí, la represión contra todo opositor hacia su persona. Bajo esta retórica de inclusión y exclusión el pueblo se encuentra dividido en dos grupos: los verdaderos nacionalistas, que son fieles a Mohammed bin Salman, y los traidores; es decir, todo aquel que se atreva a cuestionar al príncipe heredero y, por ende, a la nación saudí. Académicos, activistas, periodistas, empresarios, miembros de la familia real, minorías religiosas y clérigos son solo algunos de los grupos que han sufrido de esta represión que, si bien ha existido a lo largo de la historia saudí, hoy alcanza niveles sin precedentes.

De este modo, en Arabia Saudita se vive una dualidad de reformas, superficiales y de represión. Este es el motivo por el cual los sectores más progresistas y grupos de derechos humanos alrededor del mundo desconfían de Mohammed bin Salman. Es importante recordar que justamente su designación como príncipe heredero tenía el papel de atraer el apoyo de los jóvenes saudíes, en un país en el que los menores de 25 años representan el $49 \%$ de la población.

Por último, la estabilidad política del príncipe heredero, también se enfrenta a las consecuencias de la crisis económica. La caída de los precios del petróleo desde 2014, la 
ineficiencia de la política de austeridad aplicadas, como la quita de subsidios y el aumento del IVA y la crisis mundial provocada por la pandemia del Covid-19 amenazan los planes modernizadores del príncipe heredero y cuestionan las promesas gubernamentales respecto de los niveles de inflación y desempleo, que parecen ser demasiado optimistas. En un país en el cual la distribución de las riquezas petroleras constituye uno de los pilares de legitimidad, que la población acepte estas medidas austeras es fundamental para la estabilidad política.

El futuro de bin Salman tiene varios desafíos por delante. Mientras sus represiones pueden desatar una confrontación directa entre la realeza y los jóvenes saudíes y su creciente personalismo creará mayores fracturas dentro de la familia real, la crisis económica parece ser aún más desafiante. El príncipe heredero debe recobrar la confianza internacional, perdida especialmente desde el año 2018 con el asesinato del periodista Yamal Jashogyi, esperando poder atraer nuevas inversiones que lo ayuden a cumplir con sus planes reformistas y sus promesas de creación de empleos y de disminución de la inflación. No obstante, el mayor peligro para bin Salman estaría representado por la creación de una oposición unificada. De momento esto parece improbable al menos dentro del país, pero una organización entre los exiliados sería un gran primer paso en la lucha contra el autoritarismo saudí.

\section{Referencias}

[1] Al-Rasheed, M. (2010). A history of Saudi Arabia. Cambridge University Press.

[2] Al-Rasheed, M. (2021). The Son King: Reform and Repression in Saudi Arabia. Oxford University Press.

[3] Baamir, A. Y. (2016). Shari'a Law in Commercial and Banking Arbitration: Law and Practice in Saudi Arabia. Routledge.

[4] Bosemberg, L. E. (1998). Arabia Saudita: tribalismo, religión, conexión con Occidente y modernización conservadora. Historia Crítica, 17, 141-175. https://doi.org/10.7440/ histcrit17.1998.08

[5] Carrión, F. (2018, 8 de julio). La madre del heredero saudí lleva tres años bajo arresto domiciliario por orden de su hijo. El Mundo. https://www.elmundo.es/ loc/2018/07/08/5b33c0aae2704e76388b464f.html

[6] Crawford, M. J. (1982). Civil War, Foreign Intervention, and the Question of Political Legitimacy: A Nineteenth-Century Sa'ūdī Qāḍī's Dilemma. International Journal of Middle East Studies, 14(3), 227-248. https://doi.org/10.1017/S0020743800051928

[7] Cuesta, J. A. L. (2018). El wahabismo. La religión como elemento legitimador del poder político en Arabia Saudí, 1744-1932. Investigaciones Históricas. Época Moderna y Contemporánea, 38, 349-376. https://doi.org/10.24197/ihemc.38.2018.349-376 
[8] Cuesta, J. A. L. (2020). El wahabismo. La religión como elemento legitimador del poder político en Arabia Saudí, 1932-2005. Investigaciones Históricas. Época Moderna y Contemporánea, 40, 589-616. https://doi.org/10.24197/ihemc.40.2020.589-616

[9] Daicz, L. y Monlezún, G. (2016). El derrumbe del precio del petróleo y sus consecuencias para América Latina. Dirección Nacional Centro de Economía Internacional; Ministerio de Relaciones Exteriores y Culto República Argentina.

[10] Galindo, A. (2006). La liberalización política como estrategia del gobierno de Arabia Saudita. En R. Cornejo (comp.), En los intersticios de la democracia y el autoritarismo. Algunos casos de Asia, África y América Latina (pp. 333-360). CLACSO.

[11] Harb, A. (2019, 25 de abril). Saudi activist Abdullah Alaoudh: MBS has no Popular Legitimacy. Middle East Eye. https:/www.middleeasteye.net/news/mbs-has-nopopular-legitimacy-saudi-activist-abdullah-alaoudh-says

[12] Harrigan, P. (2016). Riyadh. Oasis of Heritage and Vision. Medina Publishing.

[13] Hernández Martínez, D. (2020). Estructura y distribución del poder en Arabia Saudí. Principales actores, instituciones y procesos de decisión. Cuadernos de Gobierno y Administración Pública, 7(1), 15-25. https://doi.org/10.5209/cgap.65525

[14] Josua, M. (2020). What Drives Diffusion? Anti-Terrorism Legislation in the Arab Middle East and North Africa. Journal of Global Security Studies, 6(3), ogaa049. https:// doi.org/10.1093/jogss/ogaa049

[15] Kechichian, J. A. (1990). Islamic Revivalism and Change in Saudi Arabia: Juhaymān Al-utaybi's "letters" to the Saudi People. The Muslim World, 80(1), 1-16. https://doi. org/10.1111/j.1478-1913.1990.tb03478.x

[16] Nehme, M. G. (1994). Saudi Development Plans between Capitalist and Islamic Values. Middle Eastern Studies, 30(3), 632-645. https://www.jstor.org/stable/4283661

[17] Nereim, V. (2021, 30 de junio). Saudis Leaving Workforce Push Unemployment to a Five-Year Low. Bloomberg. https://www.bloomberg.com/news/articles/2021-06-30/ saudi-arabia-s-jobless-rate-falls-in-progress-for-crown-prince

[18] Rich, B. (2018). Authoritarian Reformism in Saudi Arabia. E-International Relations. https://www.e-ir.info/pdf/74862

[19] Silverfarb, D. (1982). Great Britain, Iraq, and Saudi Arabia: The Revolt of the Ikhwan, 1927-1930. The International History Review, 4(2), 222-248. https://doi.org/1 $0.1080 / 07075332.1982 .9640276$

[20] Waardenburg, J. (2019). Puritans in Arabia: the Wahhabi movement (18th-19th c.). En W. E. van Beek (ed.), The Quest for Purity (pp. 129-148). De Gruyter.

[21] Wynbrandt, J. (2010). A Brief History of Saudi Arabia. Infobase Publishing. 